\title{
Abrupt cessation of short-term continuous treatment with isosorbide dinitrate may cause a rebound increase in silent myocardial ischaemia in patients with stable angina pectoris
}

\author{
Sergey Yu Martsevich, Natalia Koutishenko, Vladimir I Metelitsa
}

\begin{abstract}
Objective-To examine by Holter electrocardiographic monitoring the effect of abruptly stopping nitrate treatment in patients with stable angina pectoris.

Patients-12 men with confirmed ischaemic heart disease and stable exertional class 3 angina (Canadian). All had episodes of horizontal or down sloping ST segment depression during 24 hour electrocardiographic monitoring. All were nitrate responders.

Design-Each patient was given isosorbide dinitrate (10-30 mg four times a day) and placebo (four times a day) for three days in a randomised crossover trial. There was a washout period of 3-5 days between the two treatment periods. Holter monitoring was performed on the third day of isosorbide dinitrate and placebo administration and on the first day of their withdrawal.

Results-When treatment with isosorbide dinitrate was stopped there was a significant increase in the total number and duration of painless episodes of myocardial ischaemia. During placebo and isosorbide dinitrate administration 8 patients had episodes of painless myocardial ischaemia whereas after isosorbide dinitrate cessation they were recorded in all 12 patients. Episodes of silent myocardial ischaemia at rest appeared in 4 patients after isosorbide dinitrate with-
\end{abstract} drawal.

Conclusion-Abrupt cessation of shortterm continuous nitrate treatment in patients with severe angina may cause a rebound increase in myocardial ischaemia which is predominantly silent.

(Heart 1996;75:447-450)

Table 1 Baseline clinical characteristics of patients

\begin{tabular}{llllll}
\hline $\begin{array}{l}\text { Case } \\
\text { no }\end{array}$ & $\begin{array}{l}\text { Age } \\
(y r)\end{array}$ & $\begin{array}{l}\text { Duration of } \\
\text { angina } \\
(y r)\end{array}$ & $\begin{array}{l}\text { Previous } \\
\text { myocardial } \\
\text { infarction }\end{array}$ & $\begin{array}{l}\text { Coronary } \\
\text { angiography }\end{array}$ & $\begin{array}{l}\text { Number of } \\
\text { occluded } \\
\text { vessels }\end{array}$ \\
\hline 1 & 62 & 26 & yes & yes & 3 \\
2 & 62 & 5 & yes & no & - \\
3 & 64 & 6 & yes & no & -2 \\
4 & 54 & 5 & no & yes & 2 \\
5 & 56 & 3 & yes & yes & 3 \\
6 & 60 & 3 & yes & yes & 3 \\
7 & 65 & 6 & yes & no & - \\
8 & 60 & 1 & yes & no & -1 \\
9 & 56 & 4 & no & yes & 1 \\
10 & 55 & 1 & no & yes & 2 \\
11 & 55 & 8 & yes & yes & 3 \\
12 & 54 & 9 & yes & yes & \\
\hline
\end{tabular}

Keywords: rebound myocardial ischaemia; isosorbide dinitrate

The nitrate withdrawal phenomenon is well known. Sudden discontinuation of prolonged industrial exposure to nitrates can lead to chest pain, myocardial infarction, and even sudden death. ${ }^{12}$ The clinical significance of this phenomenon is still unclear. Few studies have investigated the risk of abrupt nitrate cessation in a clinical setting.

We have examined, by means of Holter electrocardiographic monitoring, the effect of abruptly stopping nitrates in patients with stable angina.

\section{Patients and methods}

\section{PATIENTS}

We studied 12 men aged 54-65 years (mean 59) with ischaemic heart disease and class 3 (Canadian) stable exertional angina pectoris who had 3-10 episodes of angina each day. All patients had a positive exercise treadmill test with typical angina attacks and horizontal or downsloping ST segment depression of more than $1 \mathrm{~mm} 0.08 \mathrm{~s}$ after the J point; episodes of ST segment depression of more than $1 \mathrm{~mm}$ lasting more than a minute during 24 hour Holter electrocardiographic monitoring; a positive response to isosorbide dinitrate defined as increase in the duration of the exercise treadmill test of $\geqslant 2$ minutes two hours after a single dose (compared with a control exercise test). Table 1 shows the patients' main clinical characteristics.

\section{STUDY DESIGN}

The study was approved by the institutional ethics committee. All patients gave written informed consent. The study protocol is shown in fig 1 . All antianginal medications were stopped at least 10 days before the study. During the washout period patients performed several exercise treadmill tests to assess the stability of exercise tolerance and to adjust the single effective dose of isosorbide dinitrate (see above).

The study was randomised, single blind, placebo controlled, and crossover in design. One group of patients $(n=6)$ started with isosorbide dinitrate (four times a day) for three days, followed by a washout period of 3-5 days, and then received placebo for three days. The other group $(n=6)$ followed the same 


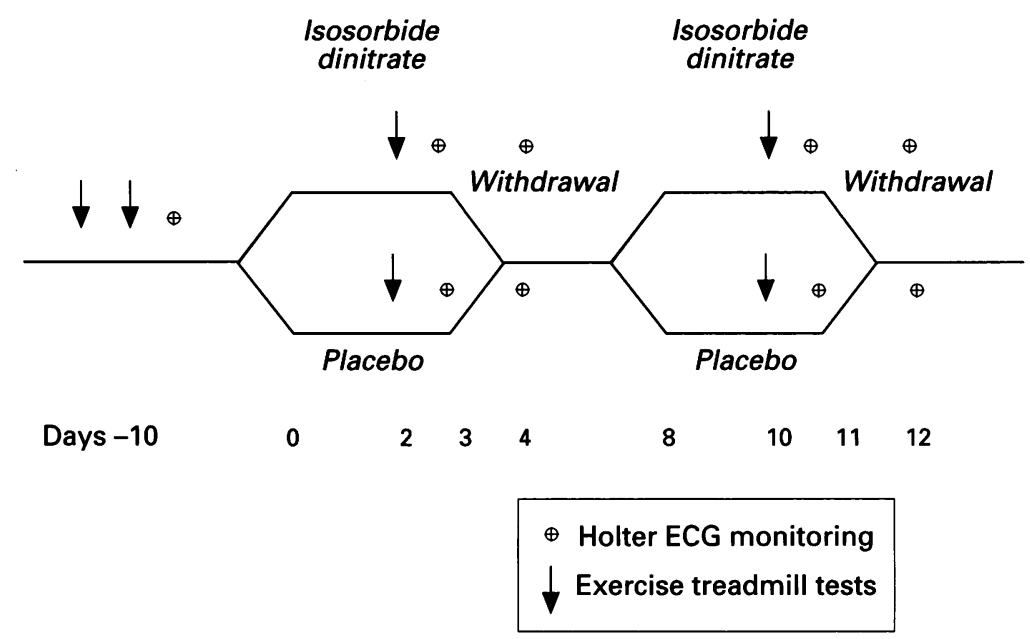

Figure 1 Study design.

Table 2 Exercise treadmill test duration (seconds) (mean (SE)) during isosorbide dinitrate and placebo administration in 12 patients.

\begin{tabular}{|c|c|c|c|c|}
\hline & \multicolumn{2}{|l|}{ Placebo } & \multicolumn{2}{|c|}{ Isosorbide dinitrate } \\
\hline & 0900 & 1630 & 0900 & 1630 \\
\hline $\begin{array}{l}\text { Exercise duration to the onset } \\
\text { of angina }\end{array}$ & $241(18)$ & $228(13)$ & $345(32)^{\star \star}$ & $264(19)$ \\
\hline $\begin{array}{l}\text { Exercise duration to the development } \\
\text { of angina of moderate severity }\end{array}$ & $290(22)$ & $270(16)$ & $414(38)^{\star \star}$ & $313(22)$ \\
\hline $\begin{array}{l}\text { Exercise duration to } S T \\
\text { segment depression }=1.0 \mathrm{~mm}\end{array}$ & $235(19)$ & $213(17)$ & $328(42)^{\star}$ & $248(28)$ \\
\hline
\end{tabular}

${ }^{\star} \mathrm{P}<0.05 ;{ }^{\star \star} \mathrm{P}<0.01$ versus placebo.

test. During electrocardiographic monitoring each patient was asked to follow their regular pattern of daily activity and to note any episodes of angina.

The recordings were analysed blindly by a doctor who was unaware of the sequence of drug/placebo administration. A computerbased scanning system (model 750A Innovator, Del Mar Avionics) was used. An ischaemic event was defined as a ST segment depression of more than $1.0 \mathrm{~mm} 0.08 \mathrm{~s}$ after the $\mathrm{J}$ point lasting at least one minute.

EXERCISE TREADMILL TESTS

To prove the efficacy of isosorbide dinitrate during continuous administration two exercise treadmill tests were performed on the second day of each treatment regimen at 0900 (two hours after the morning dose) and at 1630 ( 4.5 hours after the afternoon dose). The protocol of the exercise treadmill test has been described elsewhere. ${ }^{34}$

\section{STATISTICAL ANALYSIS}

The results are presented as mean (SE). To exclude any carry-over effects the data were compared initially by the analysis of variance. The significance of differences between isosorbide dinitrate and placebo was assessed by Student's paired $t$ test. P values of less than 0.05 were regarded as significant.

schedule but started with placebo. Treatment was stopped abruptly: the last dose was given at 1200 noon.

The single effective dose of isosorbide dinitrate was $10 \mathrm{mg}$ in 10 patients, $20 \mathrm{mg}$ in one patient, and $30 \mathrm{mg}$ in one patient. Owing to the lack of placebo identical to isosorbide dinitrate we were unable to perform the study in a double blind manner.

HOLTER ELECTROCARDIOGRAPHIC MONITORING Two channel Holter 24 hour electrocardiographic monitoring was performed on the third day of isosorbide dinitrate and placebo administration and on the first day of their withdrawal. We used a model 447 portable Holter monitor (Del Mar Avionics, USA). The leads were bipolar and attached to the sites where maximal ST segment deviations had been obtained during exercise treadmill

Figure 2 Duration of the episodes of painful (closed bars) and silent (open bars) myocardial ischaemia (minutes) during 24 hour Holter during 24 hour Holter
electrocardiographic monitoring in 12 patients. $T R$, treatment; $W D$, withdrawal. Significance of difference compared with placebo for silent placebo for silent myocardial isc
$* P<0.05$

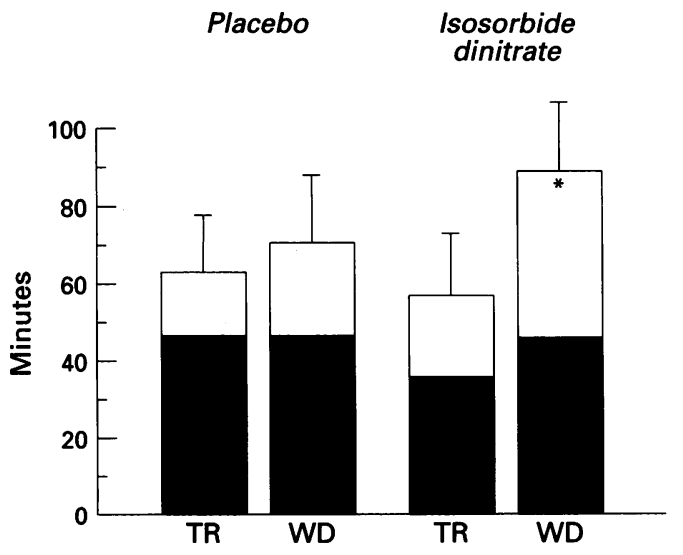

Results

ISOSORBIDE DINITRATE AND EXERCISE

TOLERANCE

Two hours after isosorbide dinitrate administration (at 0900) during continuous treatment there were significant increases (in comparison with placebo) in exercise duration to the onset of angina, to the development of angina of moderate severity, and to ST segment depression $=1 \mathrm{~mm}$. At 4.5 hours (at 1630) after the afternoon dose, however, improvement in exercise tolerance was slight and not statistically significant (table 2 ).

EPISODES OF MYOCARDIAL ISCHAEMIA

During regular isosorbide dinitrate administration there was only a tendency towards reduction in the total number of the episodes of myocardial ischaemia and their duration compared with placebo administration. When isosorbide dinitrate was abruptly stopped the number of episodes of myocardial ischaemia and their duration increased significantly. This increase was evident only for painless episodes of myocardial ischaemia (fig 2 , table 3 ).

Values for maximal ST segment depression during placebo and isosorbide dinitrate administration were substantially higher for painful episodes of myocardial ischaemia than for painless ones (table 4). The magnitude of silent ST segment depression tended to decrease during isosorbide dinitrate administration. Cessation of isosorbide dinitrate caused a significant increase in the magnitude of ST segment depression during silent (but not painful) episodes of myocardial ischaemia. 
Table 3 Number of episodes of myocardial ischaemia during 24 hour Holter electrocardiographic monitoring in 12 patients (mean (SE))

\begin{tabular}{llllll}
\hline & \multicolumn{2}{l}{ Placebo } & & \multicolumn{2}{l}{ Isosorbide dinitrate } \\
\cline { 2 - 3 } & Administration & Withdrawal & & Administration & Withdrawal \\
\hline All & $6 \cdot 8(1 \cdot 1)$ & $7 \cdot 1(1 \cdot 3)$ & & $6 \cdot 2(1 \cdot 0)$ & $9 \cdot 0(1 \cdot 3)^{\star}$ \\
Painful & $4 \cdot 6(0 \cdot 8)$ & $4 \cdot 3(0 \cdot 7)$ & & $4 \cdot 1(0 \cdot 7)$ & $4 \cdot 7(0.9)$ \\
Silent & $2 \cdot 2(0 \cdot 8)$ & $2 \cdot 8(0 \cdot 9)$ & & $2 \cdot 1(0 \cdot 6)$ & $4 \cdot 3(0 \cdot 1)^{\star}$ \\
\hline
\end{tabular}

$\star P<0.05$ versus placebo.

Table 4 Maximal ST segment depression values ( $\mathrm{mm}$ ) during 24 hour Holter electrocardiographic monitoring in 12 patients (mean (SE))

\begin{tabular}{lllllll}
\hline & Placebo & $\begin{array}{l}\text { Placebo } \\
\text { withdrawal }\end{array}$ & $P$ & $\begin{array}{l}\text { Isosorbide } \\
\text { dinitrate }\end{array}$ & $\begin{array}{l}\text { Isosorbide } \\
\text { dinitrate } \\
\text { withdrawal }\end{array}$ & $P$ \\
\hline $\begin{array}{c}\text { Painful } \\
\text { EMI }\end{array}$ & $3.06(0.56)$ & $3.07(0.53)$ & NS & $2.92(0.59)$ & $3.01(0.49)$ & NS \\
$\begin{array}{c}\text { Silent } \\
\text { EMI }\end{array}$ & $1.68(0.47)$ & $1.64(0.45)$ & NS & $1.33(0.35)$ & $2.16(0.29)$ & $<0.05$ \\
\hline
\end{tabular}

EMI, episodes of myocardial ischaemia.

INDIVIDUAL RESPONSES

There were silent episodes of myocardial ischaemia during placebo administration in nine of the 12 patients. During continuous isosorbide dinitrate administration silent episodes of myocardial ischaemia persisted in seven patients. After cessation of isosorbide dinitrate all 12 patients had silent episodes of myocardial ischaemia.

Silent myocardial ischaemia at rest was not registered in any patient during placebo administration, after its withdrawal, or during isosorbide dinitrate administration. However, after isosorbide dinitrate cessation episodes of silent myocardial ischaemia at rest were found in four patients.

\section{Discussion}

The results of the present study show that abrupt discontinuation of short-term continuous isosorbide dinitrate treatment in patients with severe stable angina pectoris can exacerbate myocardial ischaemia. The rebound effect after nitrate cessation was first described in people who had been exposed to high doses of nitrates in the munitions industry. ${ }^{25}$ Although clinical data on the possibility of nitrate rebound are scarce, ${ }^{6}$ some studies report evidence of signs of a nitrate rebound phenomenon in clinical setting.

Thus, Georgopoulos et al found an increase in the number of anginal attacks with a corresponding increase in consumption of glyceryl trinitrate in several patients during the first two days after completion of the 14 days of continuous transdermal glyceryl trinitrate treatment. ${ }^{7}$ In our previous study we detected a tendency towards an increase in exercise induced ST segment depression an hour after the removal of a single dose of transdermal glyceryl trinitrate. ${ }^{8}$ Rehnqvist et al described the appearance of severe angina necessitating admission to hospital in three of 32 patients after abrupt withdrawal of controlled-release isosorbide-5-mononitrate, although they were not sure that this was true rebound phenomenon and not simply the result of the withdrawal of beneficial treatment. ${ }^{9}$

The possibility of a nitrate withdrawal phe- nomenon was also revealed in several studies of intermittent treatment with transdermal glyceryl trinitrate. DeMots et al described the appearance of or increased frequency of nocturnal anginal attacks in nine of 206 patients receiving intermittent treatment with transdermal glyceryl trinitrate. ${ }^{10}$ They described the so-called "time-zero" effect which was manifested by the ability of a placebo group to exercise longer to the development of angina than an active treatment group at the end of the drug-free interval. Ferratini et al observed an increase in the number of angina attacks at night after the removal of transdermal glyceryl trinitrate in six out of 10 patients. ${ }^{11}$ Freedman et al described a tendency towards an increase in the duration of ischaemia during the nitrate-free interval in patients receiving transdermal glyceryl trinitrate intermittently for a week. $^{12}$

Byer and Fung recently performed a special experimental study to establish the mechanisms of nitrate rebound and found that abrupt (but not graded) withdrawal of a 90 minute intravenous infusion of glyceryl trinitrate caused a rebound increase left ventricular end diastolic pressure. They concluded that rebound depends mainly on the degree of neurohormonal activation during drug administration and the rate of its disappearance after administration stops. ${ }^{13}$

Many believe that a nitrate rebound phenomenon is unlikely under clinical conditions ${ }^{14} 15$ because many studies have not found any evidence of a deterioration in clinical condition during nitrate withdrawal. Blasini et al showed that stopping isosorbide dinitrate after induction of tolerance to medication did not cause any withdrawal phenomenon. ${ }^{16}$ Rudolph et al found no evidence of rebound during intermittent treatment with oral isosorbide dinitrate and isosorbide-5-mononitrate and with transdermal glyceryl trinitrate patches. ${ }^{14}$ Several investigators were unable to find evidence of a nitrate rebound phenomenon when different types of treatment with intermittent transdermal glyceryl trinitrate were used. ${ }^{17-20}$

Our study shows that abrupt cessation of continuous isosorbide dinitrate treatment may lead to the appearance or exacerbation of silent myocardial ischaemia. This was established by increases in the number and duration of the episodes of silent myocardial ischaemia and in the maximal ST segment depression values during silent episodes of myocardial ischaemia after nitrate treatment was stopped. Others have warned about the possibility of exacerbating episodes of silent myocardial ischaemia during nitrate-free periods though they did not observe this phenomenon in their studies. ${ }^{21}$

Despite clear evidence of exacerbation of episodes of painless myocardial ischaemia after nitrate cessation there were no real signs of deterioration of patients' clinical condition: the number of symptomatic episodes of myocardial ischaemia (that is, the number of angina attacks) during the period of nitrate withdrawal was not substantially different from that during the period of placebo with- 
drawal. This fact may partially explain the discrepancies between the results of our study and the studies that have not found clinically relevant rebound.

There is another explanation for the size of the rebound after nitrate withdrawal that was found in the present study. Most patients included in the studies which did not find any signs of nitrate withdrawal phenomenon had moderately severe angina and/or were being treated with concomitant antianginal agents $(\beta$ adrenoblockers or calcium antagonists). ${ }^{19-21}$ This was not the case in our study where all patients had class 3 angina, lower exercise tolerance, and frequent episodes of angina; no patient received any additional antianginal treatment during the study. It can be concluded, therefore, that the withdrawal phenomenon is far more likely to occur in patients with advanced ischaemic heart disease and severe angina pectoris: it may not be a problem in patients with less severe angina.

There could be a relation between the rebound phenomenon and the development of tolerance to nitrates, although this is not yet established. ${ }^{13}$ We can speculate that because the effect of isosorbide dinitrate on ischaemic episodes was slight tolerance to nitrates had developed in our patients. However, exercise tests showed a significant increase in exercise duration two hours after drug administration so perhaps tolerance was only partial.

It is arguable whether these relatively minor changes in ischaemic profile after the end of nitrate treatment are of any clinical significance. However, many believe that silent myocardial ischaemia is an important influence on prognosis ${ }^{22}{ }^{23}$ and that treatment should aim at eliminating or decreasing painless ischaemic episodes as well as painful ones. ${ }^{24}$

\section{CLINICAL IMPLICATIONS}

Nitrates are abruptly stopped in different situations-for example, during physician-initiated drug withdrawal because tolerance or side effects have developed or when a patient does not comply with the treatment regimen. It may also occur when nitrates are prescribed intermittently. The results of our study suggests that the dose should be tapered if continuous treatment with nitrates has to be stopped. We recommend that cautious attention is paid to all forms of intermittent nitrate treatment in patients with severe angina pectoris.

1 Abrams J. Nitrate tolerance and dependence. Am Heart $\mathcal{F}$ 1980;99:113-23.
2 Morton WE. Occupational habituation to aliphatic nitrates and the withdrawal hazards of coronary disease and hypertension. F Occup Med 1977;19:197-200.

3 Martsevich SY, Koutishenko N, Metelitsa VI. Withdrawal phenomenon after abrupt cessation of nifedipine in stable phenomenon after abrupt cessation of nifedipine
angina pectoris. Int $\mathcal{F}$ Cardiol 1993;42:298-301.

4 Metelitsa VI, Martsevich SY, Kozyreva MP, Slastnikova ID. Enhancement of the efficacy of isosorbide dinitrate ID. Enhancement of the efficacy of isosorbide dinitrate
by captopril in stable angina pectoris. Am f Cardiol 1992; 6y captop 6 : $291-6$.

5 Klock JC. Nonocclusive coronary disease after chronic exposure to nitrates: evidence for physiologialnitrate exposure to nitrates: evidence for phy
dependence. Am Heart $\mathcal{f} 1975 ; 89: 510-3$.

6 Morton W. Nitrate therapy in stable angina pectoris. $N$ Engl F Med 1988;318:119.

7 Georgopoulos AJ, Markis A, Georgiadis H. Therapeutic efficacy of new system containing nitroglycerin in patients with angina pectoris. Eur $f$ Clin Pharmacol 1982;22:481-5.

8 Metelitsa VI, Martsevich SY, Piotrovskii VK, Ryabokon OS, Blagodatskikh SV. New transdermal and transmucosal nitroglycerin delivery systems in patients with ischaemic heart disease. Eur $\mathcal{f}$ Clin Pharmacol 1987;32: 5-10.

9 Rehnqvist N, Olsson G, Engvall J, Rosenqvist U, Nyberg $\mathrm{G}$, Aberg A, et al. Abrupt withdrawal of isosorbide-5mononitrate in Durules (Imdur) after long term treat-
ment in patients with stable angina pectoris. Eur Heart $\mathcal{f}$ 1988;9:1339-47.

10 DeMots H, Glasser SP. Intermittent transdermal nitroglycerin therapy in the treatment of chronic stable angina. $f$ Am Coll Cardiol 1989;13:786-93.

11 Ferratini M, Pierelli S, Merlini P, Silva P, Pollavini G. Intermittent transdermal nitroglycerin monotherapy in stable exercise-induced angina: a comparison with a in stable exercise-induced angina: a comparison with
tinuous schedule. Eur Heart $\mathcal{F}$ 1989;10:998-1002.

12 Freedman SB, Daxini BV, Noyce D, Kelly DT. Intermittent transdermal nitrates do not improve ischemia in patients taking beta-blockers or calcium antagonists:
potential role of rebound ischemia during the nitrate-free potential role of rebound ischemia during

13 Bauer JA, Fung HL. Effect of apparent elimination half-life of nitroglycerin-induced hemodynamic rebound in of nitroglycerin-induced hemodynamic rebound in

14 Rudolph W, Dirschinger J, Kraus F, Reiniger G, Hall D Nitrate therapy in patients with coronary artery diseasepreparations and doses with and without development of tolerance. Z Kardiol 1990;79(suppl 3):57-65.

15 Silber S, Vogler AC, Krause KH, Theisen K. The haemodynamic and anti-ischaemic effects of a single tablet of 80 $\mathrm{mg}$ isosorbide dinitrate in slow-release formulation and review of nitrate tolerance. Drugs 1987;33(suppl 4) 69-79.

16 Blasini R, Froer KL, Blümel G, Rudolph W. Wirkungsverlust von Isosorbiddinitrat bei Langzeitbehandung der chronischen Herizinsuffizienz. Herz 1982;7:250-8.

17 Schaer DH, Buff LA, Katz RJ. Sustained antianginal efficacy of transdermal nitroglycerin patches using an
overnight 10 -hour nitrate-free interval. $A m$ Cardiol 1988;61:46-50.

18 Scardi S, Camerini F, Pandullo C, Pollavini G, Collaborative Nitro Group. Efficacy of continuous and intermittent transdermal treatment with nitroglycerin in effort angina pectoris: a multicentric study. Int $₹$ Cardiol 1991;32:241-8.

19 Gumbrielle T, Freedman SB, Fogarty L, Ogasawara S, Sobb P, Kelly DT. Efficacy, safety and duration of nitrate-free interval to prevent tolerance to transdermal nitroglycerin in effort angina. Eur Heart $f$ 1992;13:671-8.

20 nitroglycerin in effort angina. Eur Heart $\mathcal{f}$ 1992;13:671-8. Härkönen $\mathrm{R}$, et al. Acute and long-term effects on myocardial ischemia of intermittent and continuous myocardial ischemia of intermittent and continuous transdermal nitrate therapy

21 Cowan JC, Bourke JP, Reid DS, Julian DG. Prevention of tolerance to nitroglycerin patches by overnight removal. tolerance to nitroglycerin pat

22 Gottlieb SO, Gottlieb SH, Achuff SC, Baumgardner R Mellits ED, Weisfeldt ML, Gerstenblith G. Silent ischemia in Holter monitoring predicts mortality in highrisk postinfarction patients. $\mathscr{f} A M A 1988 ; 259: 1030-5$

23 Kannel WB. Detection and management of patients with silent myocardial ischemia. Am Heart $\mathcal{F} 1989 ; 117: 221-6$.

24 Lim R, Dyke LJ, Dymond DS. Suppression of inducible painless myocardial ischemia by conventional medical therapy: effect on short-term outcome and left ventricular systolic function. Am $\mathcal{F}$ Cardiol 1994;73:26B-9B. 